American Journal of Applied Sciences 6 (3): 387-392, 2009

ISSN 1546-9239

(C) 2009 Science Publications

\title{
Economic Ordering Quantity Model with Lead Time Reduction and Backorder Price Discount for Stochastic Demand
}

\author{
Ming-Cheng Lo \\ Ching Yun University, 229, Chien-Hsin Road, Jung-Li 320, Taiwan, Republic of China
}

\begin{abstract}
This study considered reorder point on the continuous review inventory model under controllable lead time with mixture of backorder price discounts and partial lost sales. We developed a continuous review inventory model where the lead time, the order quantity, backorder discount and safety factor were considered as the decision variables of a mixture of backorders and lost sales inventory model. The objective was to minimize the expected total annual cost with respect to related decision variables. The purpose model with lead time demand distribution was unknown. The author applies a minimax distribution free procedure to find the optimal solution and numerical example was included to illustrate the solution procedure of the proposed algorithms.
\end{abstract}

Key words: Lead time, backorder price discounts, reorder point, minimax distribution free procedure

\section{INTRODUCTION}

It is important to manage inventory quantity for modern enterprises. Although the time condensation will predictably raise the cost, a customer will pay remuneration to the supplier who can provide its product faster and more firm than the competition and the price payment may be praiseworthy. Silver and Peterson ${ }^{[18]}$ defined the replenishment lead time as the time beyond from the moment at which it is decided to place an order, until it is physically on the shelf to satisfy customer demands. While Naddor ${ }^{[12]}$, Silver and Peterson ${ }^{[18]}$ consider that lead time can be a constant or a random variable, it is often treated as a prescribed parameter and not controllable. However, lead time can be reduced at extra cost and shorter lead time is the primary driver to achieving customer satisfaction in successful TBM operations ${ }^{[1]}$. The benefits resulting from reduced lead time include lower cost, less waste and less obsolescence, greater flexibility to response to change, closely linked organization priorities to customers' needs, improved service, quality and reliability and substantially accelerated supply system improvements $^{[1]}$. Tersine ${ }^{[20]}$ and Vollmann et al. ${ }^{[21]}$ attributed the replenishment lead time mostly to manufacturing considerations and addressed some guidelines for its reduction. Liao and Shyu ${ }^{[5]}$ suggested that lead time could be decomposed into $\mathrm{n}$ components each having a different crashing cost for reduction. Ben-Daya and Raouf ${ }^{[2]}$ widespread the Liao and Shyu ${ }^{[5]}$ model by considering both lead time and the order quantity as decision variables. It is related ${ }^{[2,4,10,13]}$ are all focus on the reorder point or safety factor as a decision variable.

We solve the problem by applying the minimax distribution-free approach, originally disseminated by Gallego and Moon ${ }^{[3]}$. Moon and Choi ${ }^{[8]}$ and Moon and Yun ${ }^{[9]}$ apply distribution-free approach to solving the purpose models. Ouyang and $\mathrm{Wu}^{[15]}$ relaxed the assumption on the form of cumulative distribution function of the lead time demand and applied the minimax distribution free procedure to solve the problem. Moon and Silver ${ }^{[1]}$ and Silver and Moon ${ }^{[19]}$ study a single period replenishment problem under the distribution free situation. On the other hand, this study considers a continuous review inventory system in which the lead time is controllable and can be decomposed into several components each having a crashing cost function. In addition, shortage is permitted and the total amount of stockout is a combination of backorder and lost sale. It is further assumed that the patient customers with outstanding orders during the shortage period are offered a backorder price discount and consequently the backorder ratio is proportional to the magnitude of this discount ${ }^{[16]}$. Since the shortage cost is explicitly included, the reorder point is also treated as a decision variable in this study ${ }^{[10]}$.

There is a form of lead time demand that assumes a distribution free in the study. In this model, the objectives are to simultaneously optimize the order quantities, back order discounts, reorder points and lead

Corresponding Author: Ming-Cheng Lo, Department of Business Administration, Ching Yun University, 229, Chien-Hsin Road, Jung-Li, 320, Taiwan, Republic of China 
times such that the total expected annual costs are minimized. Finally, a particular case is deduced, which has been previously available and a numerical illustrative example is added.

\section{DISTRIBUTION FREE MODEL}

In a distribution free model, the lead time demand assumption is relaxed to any distribution function by only assuming that the distribution function $\mathrm{F}$ belongs to the class $\Omega$ with finite mean $\mu \mathrm{L}$ and variance $\sigma^{2} \mathrm{~L}$. Again, the expected total annual cost of the distribution free model can be expressed as $\operatorname{EACD}\left(\mathrm{Q}, \pi_{\mathrm{x}}\right.$, $\mathrm{k}, \mathrm{L})=\mathrm{OC}+\mathrm{HC}+\mathrm{SC}+\mathrm{CC}$. While the expected ordering and expected crashing costs are of the same forms as those of the normal demand model, the expected shortage at the end of a cycle can be expressed by $\mathrm{B}(\mathrm{r})=\mathrm{E}[\mathrm{X}-\mathrm{r}]^{+}$and the corresponding expected total annual holding cost is:

$$
\mathrm{HC}=\mathrm{h}\left\{\frac{\mathrm{Q}}{2}+\mathrm{k} \sigma \sqrt{\mathrm{L}}+(1-\beta) \mathrm{E}[\mathrm{X}-\mathrm{r}]^{+}\right\}
$$

The expected annual shortage cost is:

$$
\mathrm{SC}=\frac{\mathrm{D}}{\mathrm{Q}}\left[\beta \pi_{\mathrm{x}}+(1-\beta) \pi_{0}\right] \mathrm{E}[\mathrm{X}-\mathrm{r}]^{+}
$$

Therefore, the expected total annual cost for distribution free model can be presented by:

$$
\begin{aligned}
\operatorname{EACD}(\mathrm{Q}, & \pi \mathrm{x}, \mathrm{k}, \mathrm{L}) \\
= & \mathrm{A} \frac{\mathrm{D}}{\mathrm{Q}}+\mathrm{h}\left[\frac{\mathrm{Q}}{2}+\mathrm{k} \sigma \sqrt{\mathrm{L}}+(1-\beta) \mathrm{E}[\mathrm{X}-\mathrm{r}]^{+}\right] \\
+ & \frac{\mathrm{D}}{\mathrm{Q}}\left[\beta \pi_{\mathrm{x}}+(1-\beta) \pi_{0}\right] \mathrm{E}[\mathrm{X}-\mathrm{r}]^{+} \\
+ & \frac{\mathrm{D}}{\mathrm{Q}}\left[\mathrm{a}_{\mathrm{i}}\left(\mathrm{L}_{\mathrm{i}-1}-\mathrm{L}\right)+\sum_{\mathrm{j}=1}^{\mathrm{i}-1} \mathrm{a}_{\mathrm{j}}\left(\mathrm{T}_{\mathrm{j}}-\mathrm{t}_{\mathrm{j}}\right)\right] \\
= & \mathrm{A} \frac{\mathrm{D}}{\mathrm{Q}}+\mathrm{h}\left[\frac{\mathrm{Q}}{2}+\mathrm{k} \sigma \sqrt{\mathrm{L}}+(1-\beta) \sigma \sqrt{\mathrm{L} \Psi(\mathrm{k})}\right] \\
+ & \frac{\mathrm{D}}{\mathrm{Q}}\left[\beta \pi_{\mathrm{x}}+(1-\beta) \pi_{0}\right] \mathrm{E}[\mathrm{X}-\mathrm{r}]^{+} \\
& +\frac{\mathrm{D}}{\mathrm{Q}}\left[\mathrm{a}_{\mathrm{i}}\left(\mathrm{L}_{\mathrm{i}-1}-\mathrm{L}\right)+\sum_{\mathrm{j}=1}^{\mathrm{i}-1} \mathrm{a}_{\mathrm{j}}\left(\mathrm{T}_{\mathrm{j}}-\mathrm{t}_{\mathrm{j}}\right)\right] \\
& \quad \text { for } \mathrm{L}_{\mathrm{i}}<\mathrm{L} \leq \mathrm{L}_{\mathrm{i}-1}
\end{aligned}
$$

Since the form of the lead time demand distribution is not known, the minimax criterion is applied to find the least favorite distribution function in $\Omega$ for each $(\mathrm{Q}$, $\left.\pi_{\mathrm{x}}, \mathrm{k}, \mathrm{L}\right)$ and then find the optimal values of $\mathrm{Q}^{*}, \pi_{\mathrm{x}}{ }^{*}$, $\mathrm{k}^{*}$ and $\mathrm{L}$ that minimize the expected total annual cost. In mathematical symbolization, the problem under investigation can be expressed as:

$$
\min _{\mathrm{Q}>0, \mathrm{~L}>0} \max _{\mathrm{F} \in \Omega} \operatorname{EACD}(\mathrm{Q}, \pi \mathrm{x}, \mathrm{k}, \mathrm{L})
$$

Gallego and Moon $^{[3]}$ proved that the following inequality holds for any $\mathrm{F} \in \Omega$ :

$$
\mathrm{E}(\mathrm{X}-\mathrm{r})^{+} \leq \frac{1}{2}\left[\sqrt{\sigma^{2} \mathrm{~L}+(\mathrm{r}-\mu \mathrm{L})^{2}}-(\mathrm{r}-\mu \mathrm{L})\right]
$$

Substituting $r=\mu \mathrm{L}+k \sigma \sqrt{\mathrm{L}}$ into the model, the problem can be reduced to minimize:

$\operatorname{EACD}(\mathrm{Q}, \pi \mathrm{x}, \mathrm{k}, \mathrm{L})$

$=\mathrm{A} \frac{\mathrm{D}}{\mathrm{Q}}+\mathrm{h}\left[\frac{\mathrm{Q}}{2}+\mathrm{k} \sigma \sqrt{\mathrm{L}}\right]$

$+\frac{1}{2}\left\{\mathrm{~h}\left[1-\frac{\beta_{0}}{\pi_{0}} \pi_{\mathrm{x}}\right]+\frac{\mathrm{D}}{\mathrm{Q}}\left[\frac{\beta_{0}}{\pi_{0}} \pi_{\mathrm{x}}^{2}+\pi_{0}-\beta_{0} \pi_{\mathrm{x}}\right]\right\}$

$\times \sigma \sqrt{L}\left(\sqrt{1+k^{2}}-k\right)+\frac{D}{Q}\left[a_{i}\left(L_{i-1}-L\right)+\sum_{j=1}^{i-1} a_{j}\left(T_{j}-t_{j}\right)\right]$

for $\mathrm{L}_{\mathrm{i}}<\mathrm{L} \leq \mathrm{L}_{\mathrm{i}-1}$

Taking the partial derivatives of $\operatorname{EACD}\left(\mathrm{Q}, \pi_{\mathrm{x}}, \mathrm{k}\right.$, $\mathrm{L})$ with respect to $\mathrm{Q}, \pi_{\mathrm{x}}, \mathrm{k}$ and $\mathrm{L}$ in each time interval $\left(\mathrm{L}_{\mathrm{i}}, \mathrm{L}_{\mathrm{i}-1}\right]$, we obtain:

$$
\begin{gathered}
\frac{\partial \operatorname{EACD}\left(\mathrm{Q}, \pi_{\mathrm{x}}, \mathrm{k}, \mathrm{L}\right)}{\partial \mathrm{Q}} \\
=-\frac{\mathrm{AD}}{\mathrm{Q}^{2}}+\frac{\mathrm{h}}{2}-\frac{\mathrm{D}}{2 \mathrm{Q}^{2}}\left[\frac{\beta_{0}}{\pi_{0}} \pi_{\mathrm{x}}^{2}+\pi_{0}-\beta_{0} \pi_{\mathrm{x}}\right] \\
\sigma \sqrt{\mathrm{L}}\left(\sqrt{1+\mathrm{k}^{2}}-\mathrm{k}\right) \\
-\frac{\mathrm{D}}{\mathrm{Q}^{2}}\left[\mathrm{a}_{\mathrm{i}}\left(\mathrm{L}_{\mathrm{i}-1}-\mathrm{L}\right)+\sum_{\mathrm{j}=1}^{\mathrm{i}-1} \mathrm{a}_{\mathrm{j}}\left(\mathrm{T}_{\mathrm{j}}-\mathrm{t}_{\mathrm{j}}\right)\right] \\
\frac{\partial \operatorname{EACD}\left(\mathrm{Q}, \pi_{\mathrm{x}}, \mathrm{k}, \mathrm{L}\right)}{\partial \pi_{\mathrm{x}}}=\frac{1}{2}\left[-\frac{\mathrm{h} \beta_{0}}{\pi_{0}}+\frac{\mathrm{D}}{\mathrm{Q}}\left(\frac{2 \beta_{0}}{\pi_{0}} \pi_{\mathrm{x}}-\beta_{0}\right)\right] \\
\sigma \sqrt{\mathrm{L}}\left(\sqrt{1+\mathrm{k}^{2}}-\mathrm{k}\right)
\end{gathered}
$$


Am. J. Applied Sci., 6 (3): 387-392, 2009

$$
\begin{aligned}
\frac{\partial \operatorname{EACD}\left(\mathrm{Q}, \pi_{\mathrm{x}}, \mathrm{k}, \mathrm{L}\right)}{\partial \mathrm{L}}= & \frac{1}{4}\left[\begin{array}{l}
\mathrm{h}\left(1-\frac{\beta_{0}}{\pi_{0}} \pi_{\mathrm{x}}\right) \\
+\frac{\mathrm{D}}{\mathrm{Q}}\left(\frac{\beta_{0}}{\pi_{0}} \pi_{\mathrm{x}}^{2}+\pi_{0}-\beta_{0} \pi_{\mathrm{x}}\right)
\end{array}\right] \\
& \sigma \mathrm{L}^{-\frac{1}{2}}\left(\sqrt{1+\mathrm{k}^{2}}-\mathrm{k}\right) \\
& +\frac{1}{2} \mathrm{hk \sigma L}^{-1 / 2}-\frac{\mathrm{D}}{\mathrm{Q}} \mathrm{a}_{\mathrm{i}}
\end{aligned}
$$

However, for fixed $\mathrm{Q}, \mathrm{k}$ and $\pi_{\mathrm{x}}, \operatorname{EACD}\left(\mathrm{Q}, \pi_{\mathrm{x}}, \mathrm{k}, \mathrm{L}\right)$ is concave in $L \in\left(\mathrm{L}_{\mathrm{i}}, \mathrm{L}_{\mathrm{i}-1}\right]$, since:

$$
\begin{aligned}
\frac{\partial \operatorname{EACD}\left(\mathrm{Q}, \pi_{\mathrm{x}}, \mathrm{k}, \mathrm{L}\right)}{\partial \mathrm{L}^{2}}=-\frac{1}{8}\left[\begin{array}{l}
\mathrm{h}\left(1-\frac{\beta_{0}}{\pi_{0}} \pi_{\mathrm{x}}\right) \\
+\frac{\mathrm{D}}{\mathrm{Q}}\left(\frac{\beta_{0}}{\pi_{0}} \pi_{\mathrm{x}}^{2}+\pi_{0}-\beta_{0} \pi_{\mathrm{x}}\right)
\end{array}\right] \\
\sigma \mathrm{L}^{-\frac{3}{2}}\left(\sqrt{1+\mathrm{k}^{2}}-\mathrm{k}\right)-\frac{1}{4} \mathrm{hk \sigma \textrm {L } ^ { - \frac { 3 } { 2 } }}<0
\end{aligned}
$$

For fixed $L \in\left(\mathrm{L}_{\mathrm{i}}, \mathrm{L}_{\mathrm{i}-1}\right]$, the determinant of Hessian matrix of $\operatorname{EACD}\left(\mathrm{Q}, \pi_{\mathrm{x}}, \mathrm{k}, \mathrm{L}\right)$ is positive definite at $\left(\mathrm{Q}^{*}\right.$, $\left.\pi_{\mathrm{x}}{ }^{*}, \mathrm{k}^{*}\right)$ as proved in Appendix. Setting equation (5) to zero and solving for $\pi_{\mathrm{x}}$, it follows that:

$$
\pi_{\mathrm{x}}^{*}=\frac{\mathrm{hQ}}{2 \mathrm{D}}+\frac{\pi_{0}}{2}
$$

Setting Eq. 4 to zero and substituting (9) into (4) to solve for $\mathrm{Q}$, it follows that:

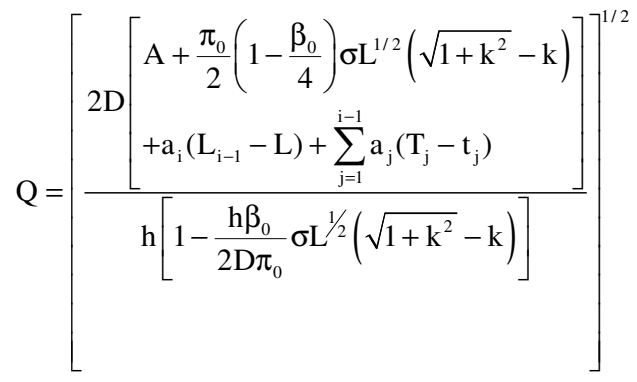

Solving for $\mathrm{k}$ by setting Eq. 6 to zero and substituting (9) into (6), we have:

$$
\frac{\mathrm{k}}{\sqrt{1+\mathrm{k}^{2}}}=1-\frac{2 \mathrm{~h}}{\left[\begin{array}{l}
\mathrm{h}\left(1-\frac{\beta_{0} \mathrm{hQ}}{2 \pi_{0} \mathrm{D}}-\frac{\beta_{0}}{2}\right) \\
+\frac{\alpha_{0} \mathrm{D}}{\mathrm{Q}}\left(\frac{\beta_{0}}{4}\left(\frac{\mathrm{hQ}}{\pi_{0} \mathrm{D}}\right)^{2}+\left(1-\frac{\beta_{0}}{4}\right)\right)
\end{array}\right]}
$$

The following algorithm is developed to find the optimal values of the order quantity, backorder price discount, safety factor and lead time for the problem under study.

Step 1: For i $=0,1,2, \ldots, \mathrm{n}$

(i) $\quad$ Set $\mathrm{k}_{\mathrm{io}}=0$

(ii) Substitute $\mathrm{k}_{\mathrm{io}}$ into (10) to evaluate $\mathrm{Q}_{\text {io }}$

(iii) Utilize $\mathrm{Q}_{\text {io }}$ to determine $\mathrm{k}_{\text {in }}$ from (11). Let $\mathrm{k}_{\mathrm{io}}=\mathrm{k}_{\text {in }}$

(iv) Repeat (ii) and (iii) until no change occurs in the values of $\mathrm{Q}_{i}$ and $\mathrm{k}_{\mathrm{i}}$. Denote the resulting solutions by $\mathrm{Q}_{\mathrm{i}}$ and $\mathrm{k}_{\mathrm{i}}$

(v) For $\mathrm{i}=0,1,2, \ldots, \mathrm{n}$, use equation (3) to compute the expected total annual cost $\operatorname{EAC}\left(\mathrm{Q}_{\mathrm{i}}, \pi_{\mathrm{xi}}, \mathrm{k}_{\mathrm{i}}, \mathrm{L}_{\mathrm{i}}\right)$

Step 2: Set $\operatorname{EACD}\left(\mathrm{Q}^{*}, \pi_{\mathrm{x}}{ }^{*}, \mathrm{k}^{*}, \mathrm{~L}^{*}\right)=\operatorname{Min}\left\{\operatorname{EACD}\left(\mathrm{Q}_{\mathrm{i}}\right.\right.$, $\left.\left.\pi_{\mathrm{xi}}, \mathrm{k}_{\mathrm{i}}, \mathrm{L}_{\mathrm{i}}\right), \mathrm{i}=0,1,2, \ldots, \mathrm{n}\right\}$.

Step 3: $\left.Q^{*}, \pi_{x}{ }^{*}, k^{*}, L^{*}\right)$ is a set of optimal solutions.

\section{NUMERICAL EXAMPLE}

Consider an inventory system with the following data: $\mathrm{D}=600$ units/year, $\mathrm{A}=\$ 200$ per order, $\mathrm{h}=\$ 20$ per unit per year, $\pi_{0}=\$ 150$ per unit, $\sigma=7$ unit/week and the lead time has three components with data shown in Table $1^{[16]}$, except that the probability distribution of the lead time demand is unknown. Apply the proposed algorithm to solve the problem for the upper bound of the backorder ratio $\beta_{0}=0.95,0.80$, $0.65,0.50,0.35$ and 0.20 . The results are summarized in Table 2.

Next, compare the performance of distribution-free approach against those of normal distribution. For example, consider the results with $\beta_{0}=0.5$. Let $\left(Q^{\mathrm{N}}\right.$, $\left.\pi_{\mathrm{x}}^{\mathrm{N}}, \mathrm{k}^{\mathrm{N}}, \mathrm{L}_{\mathrm{i}}^{\mathrm{N}}\right)=(121,77.0157,1.88,4)$ denote the optimal solution set obtained in the normal distribution case ${ }^{[13]}$ and $\operatorname{EAC}^{\mathrm{N}}\left(\bullet^{\mathrm{N}}\right)$ be the associated cost where $\mathrm{N}$ represents the normal distribution. On the other hand, let $\left(\mathrm{Q}^{\mathrm{U}}, \pi_{\mathrm{x}}^{\mathrm{U}}, \mathrm{k}^{\mathrm{U}}, \mathrm{L}_{\mathrm{i}}^{\mathrm{U}}\right)=(146,77.43,2.49,3)$ denote the optimal solution set found in the general distribution case where $\mathrm{U}$ denote the distribution free case with associated cost $\operatorname{EAC}^{\mathrm{N}}\left(\bullet^{\mathrm{U}}\right)$ and $\operatorname{EAC}^{\mathrm{N}}\left(\bullet^{\mathrm{U}}\right)$ is the annual expected cost of using $\left(\mathrm{Q}^{\mathrm{U}}, \pi_{\mathrm{x}}^{\mathrm{U}}, \mathrm{k}^{\mathrm{U}}, \mathrm{L}_{\mathrm{i}}^{\mathrm{U}}\right)$ when the real demand distribution is normal. Then the additional cost of $\operatorname{EAC}^{\mathrm{N}}\left(\bullet^{\mathrm{U}}\right)-\operatorname{EAC}^{\mathrm{N}}\left(\bullet^{\mathrm{N}}\right)=\operatorname{EAC}^{\mathrm{N}}(146,77.43,2.49$,

Table 1: Lead time data of the examples

\begin{tabular}{llll}
\hline Lead time component $\mathrm{I}$ & 1 & 2 & 3 \\
\hline Normal duration $\mathrm{T}_{\mathrm{i}}$ (days) & 20 & 20 & 16 \\
Minimum duration $\mathrm{t}_{\mathrm{i}}$ (days) & 6.0 & 6.0 & 9.0 \\
Unit fixed crashing cost $\mathrm{a}_{\mathrm{i}}$ (\$/day) & 0.4 & 1.2 & 5.0 \\
\hline
\end{tabular}


Am. J. Applied Sci., 6 (3): 387-392, 2009

Table 2: Summary of the results for example ( $\mathrm{L}_{\mathrm{i}}$ in weeks)

\begin{tabular}{|c|c|c|c|c|c|c|c|c|c|c|}
\hline \multirow[b]{2}{*}{$\beta_{0}$} & \multicolumn{5}{|c|}{ The proposed model } & \multicolumn{4}{|c|}{ The Pan and Hsiao ${ }^{[16]}$ model $(\mathrm{k}=0.85)$} & \multirow{2}{*}{$\begin{array}{l}\text { Savings } \\
(\%)((2) \\
-(1)) /(2)\end{array}$} \\
\hline & $Q^{*}$ & $\pi_{\mathrm{x}}^{*}$ & $\mathrm{k}^{*}$ & $\mathrm{~L}_{\mathrm{i}}^{*}$ & $\operatorname{EAC}(\bullet)^{(1)}$ & $\mathrm{Q}^{*}$ & $\pi_{\mathrm{x}}^{*}$ & $\mathrm{~L}_{\mathrm{i}}^{*}$ & $\operatorname{EAC}(\bullet)^{(2)}$ & \\
\hline 0.95 & 144 & 77.39 & 2.32 & 3 & 3684.90 & 208 & 77.95 & 3 & 3964.73 & 7.06 \\
\hline 0.80 & 144 & 77.40 & 2.38 & 3 & 3714.58 & 212 & 77.99 & 3 & 4019.26 & 7.58 \\
\hline 0.65 & 145 & 77.42 & 2.43 & 3 & 3743.47 & 216 & 78.03 & 3 & 4073.15 & 8.09 \\
\hline 0.50 & 146 & 77.43 & 2.49 & 3 & 3771.61 & 219 & 78.08 & 3 & 4126.41 & 8.60 \\
\hline 0.35 & 146 & 77.44 & 2.54 & 3 & 3799.06 & 223 & 78.12 & 3 & 4179.07 & 9.09 \\
\hline 0.20 & 147 & 77.45 & 2.60 & 3 & 3825.86 & 226 & 78.16 & 3 & 4231.15 & 9.58 \\
\hline
\end{tabular}

Table 3: Summary of computational results

\begin{tabular}{llllll}
\hline$\beta_{0}$ & $\begin{array}{l}\mathrm{EAC}^{\mathrm{U}} \\
\left(\mathrm{Q}^{\mathrm{U}}, \pi_{\mathrm{x}}^{\mathrm{U}}, \mathrm{k}^{\mathrm{U}}, \mathrm{L}_{\mathrm{i}}^{\mathrm{U}}\right)\end{array}$ & $\begin{array}{l}\mathrm{EAC}^{\mathrm{N}} \\
(\bullet \mathrm{U})\end{array}$ & $\begin{array}{l}\mathrm{EAC}^{\mathrm{N}} \\
(\bullet \mathrm{U})\end{array}$ & $\begin{array}{l}\operatorname{EAC}^{\mathrm{N}} \\
(\bullet \mathrm{N})\end{array}$ & $\begin{array}{l}\operatorname{EAC}^{\mathrm{N}}(\bullet \mathrm{U}) \\
/ \mathrm{EAC}^{\mathrm{N}}\left(\bullet^{\mathrm{N}}\right)\end{array}$ \\
\hline 0.95 & $(144,77.39,2.32,3)$ & 3684.90 & 3083.65 & 2932.15 & 1.052 \\
0.80 & $(144,77.40,2.38,3)$ & 3714.58 & 3063.23 & 2937.62 & 1.043 \\
0.65 & $(145,77.42,2.43,3)$ & 3743.47 & 3079.66 & 2942.81 & 1.047 \\
0.50 & $(146,77.43,2.49,3)$ & 3771.61 & 3095.93 & 2947.72 & 1.050 \\
0.35 & $(146,77.44,2.54,3)$ & 3799.06 & 3112.02 & 2952.40 & 1.054 \\
0.20 & $(147,77.45,2.60,3)$ & 3825.86 & 3127.95 & 2956.85 & 1.058 \\
\hline
\end{tabular}

$3)-\mathrm{EAC}^{\mathrm{N}}(121, \quad 77.0157, \quad 1.88, \quad 4)=\$ 3095.93-$ $2947.72=\$ 148.21$ is the largest amount that one would be willing to pay for the knowledge of the distributional form of lead time demand. This quantity can be regarded as the Expected Value of Additional Information (EVAI) ${ }^{[3]}$.

The results for $\beta_{0}=0.95,0.80,0.65,0.50,0.35$ and 0.20 in Table 3 reconfirm the robustness of distribution free approach that has been proven in recent related studies $^{[10]}$.

\section{CONCLUSIONS}

This research studies distribution free model having both mean and variance known and finite. We consider the impact of safety factor on the continuous review inventory model involving controllable lead time and backorder price discount with mixture of backorder and partial lost sales. The objective is to minimize the expected total annual cost by simultaneously optimizing order quantity, backorder price discount, safety factor and lead time. Algorithms are developed to find the optimal solutions for the model and example are provided to illustrate the procedures of the algorithms. We may obtain one conclusion when the upper bound of the backorder ratio $\beta_{0}$ increases, backorder ratio $\beta$ and safety factor and total expected annual cost decreases. If the buyer permits seller bigger backorder price discount, will be allowed to promote the service level and to reduce total expected annual cost.

\section{APPENDIX}

The Hessian matrix $\mathrm{H}$ of $\operatorname{EACD}\left(\mathrm{Q}, \pi_{\mathrm{x}}, \mathrm{k}, \mathrm{L}\right)$ for $\mathrm{a}$ given value of $\mathrm{L}$ is:

$$
H=\left[\begin{array}{cc}
\frac{\partial^{2} \operatorname{EACD}\left(\mathrm{Q}, \pi_{\mathrm{x}}, \mathrm{k}, \mathrm{L}\right)}{\partial \mathrm{Q}^{2}} & \frac{\partial^{2} \mathrm{EACD}\left(\mathrm{Q}, \pi_{\mathrm{x}}, \mathrm{k}, \mathrm{L}\right)}{\partial \mathrm{Q} \partial \pi_{\mathrm{x}}} \\
\frac{\partial^{2} \mathrm{EACD}\left(\mathrm{Q}, \pi_{\mathrm{x}}, \mathrm{k}, \mathrm{L}\right)}{\partial \pi_{\mathrm{x}} \partial \mathrm{Q}} & \frac{\partial^{2} \mathrm{EACD}\left(\mathrm{Q}, \pi_{\mathrm{x}}, \mathrm{k}, \mathrm{L}\right)}{\partial \pi_{\mathrm{x}}^{2}} \\
\frac{\partial^{2} \mathrm{EACD}\left(\mathrm{Q}, \pi_{\mathrm{x}}, \mathrm{k}, \mathrm{L}\right)}{\partial \mathrm{k} \partial \mathrm{Q}} & \frac{\partial^{2} \mathrm{EACD}\left(\mathrm{Q}, \pi_{\mathrm{x}}, \mathrm{k}, \mathrm{L}\right)}{\partial \mathrm{k} \partial \pi_{\mathrm{x}}} \\
\frac{\partial^{2} \mathrm{EACD}\left(\mathrm{Q}, \pi_{\mathrm{x}}, \mathrm{k}, \mathrm{L}\right)}{\partial \mathrm{Q} \partial \mathrm{k}} \\
\frac{\partial^{2} \mathrm{EACD}\left(\mathrm{Q}, \pi_{\mathrm{x}}, \mathrm{k}, \mathrm{L}\right)}{\partial \pi_{\mathrm{x}} \partial \mathrm{k}} \\
\frac{\partial^{2} \mathrm{EACD}\left(\mathrm{Q}, \pi_{\mathrm{x}}, \mathrm{k}, \mathrm{L}\right)}{\partial \mathrm{k}_{\mathrm{x}}^{2}}
\end{array}\right]
$$

Where:

$$
\begin{aligned}
& \frac{\partial^{2} \operatorname{EACD}\left(\mathrm{Q}, \pi_{\mathrm{x}}, \mathrm{k}, \mathrm{L}\right)}{\partial \mathrm{Q}^{2}}=\frac{2 \mathrm{AD}}{\mathrm{Q}^{3}}+\frac{\mathrm{D}}{\mathrm{Q}^{3}}\left[\frac{\beta_{0}}{\pi_{0}} \pi_{\mathrm{x}}^{2}+\pi_{0}-\beta_{0} \pi_{\mathrm{x}}\right] \\
& \sigma \sqrt{\mathrm{L}}\left(\sqrt{1+\mathrm{k}^{2}}-\mathrm{k}\right) \\
& +\frac{2 D}{Q^{3}}\left[a_{i}\left(L_{i-1}-L\right)+\sum_{j=1}^{i-1} a_{j}\left(T_{j}-t_{j}\right)\right] \\
& \frac{\partial^{2} \operatorname{EACD}\left(\mathrm{Q}, \pi_{\mathrm{x}}, \mathrm{k}, \mathrm{L}\right)}{\partial \pi_{\mathrm{x}}^{2}}=\frac{2 \mathrm{D} \beta_{0}}{\mathrm{Q} \pi_{0}} \sigma \sqrt{\mathrm{L}} \Psi(\mathrm{k}) \\
& \frac{\partial^{2} \operatorname{EACD}\left(\mathrm{Q}, \pi_{\mathrm{x}}, \mathrm{k}, \mathrm{L}\right)}{\partial \mathrm{k}^{2}}=\frac{1}{2}\left[\begin{array}{l}
\mathrm{h}\left(1-\frac{\beta_{0}}{\pi_{0}} \pi_{\mathrm{x}}\right) \\
+\frac{\mathrm{D}}{\mathrm{Q}}\left(\frac{\beta_{0}}{\pi_{0}} \pi_{\mathrm{x}}^{2}+\pi_{0}-\beta_{0} \pi_{\mathrm{x}}\right)
\end{array}\right] \\
& \sigma \sqrt{\mathrm{L}}\left(\sqrt{1+\mathrm{k}^{2}}\right)^{-3 / 2} \\
& \frac{\partial^{2} \operatorname{EACD}\left(\mathrm{Q}, \pi_{\mathrm{x}}, \mathrm{k}, \mathrm{L}\right)}{\partial \mathrm{Q} \partial \pi_{\mathrm{x}}}=\frac{\partial^{2} \operatorname{EACD}\left(\mathrm{Q}, \pi_{\mathrm{x}}, \mathrm{k}, \mathrm{L}\right)}{\partial \pi_{\mathrm{x}} \partial \mathrm{Q}} \\
& =-\frac{\mathrm{D}}{2 \mathrm{Q}^{2}}\left[\frac{2 \beta_{0}}{\pi_{0}} \pi_{\mathrm{x}}-\beta_{0}\right] \sigma \sqrt{\mathrm{L}}\left(\sqrt{1+\mathrm{k}^{2}}-\mathrm{k}\right) \\
& \frac{\partial^{2} \operatorname{EACD}\left(\mathrm{Q}, \pi_{\mathrm{x}}, \mathrm{k}, \mathrm{L}\right)}{\partial \mathrm{k} \partial \pi_{\mathrm{x}}}=\frac{\partial^{2} \operatorname{EACD}\left(\mathrm{Q}, \pi_{\mathrm{x}}, \mathrm{k}, \mathrm{L}\right)}{\partial \pi_{\mathrm{x}} \partial \mathrm{k}} \\
& \left.=\frac{1}{2}\left[\mathrm{~h} \frac{\beta_{0}}{\pi_{0}}-\frac{\mathrm{D}}{\mathrm{Q}}\left(\frac{2 \beta_{0}}{\pi_{0}} \pi_{\mathrm{x}}-\beta_{0}\right)\right] \sigma \sqrt{\mathrm{L}}\left(1-\frac{\mathrm{k}}{\sqrt{1+\mathrm{k}^{2}}}\right)\right)
\end{aligned}
$$


and

$\frac{\partial^{2} \operatorname{EACD}\left(\mathrm{Q}, \pi_{\mathrm{x}}, \mathrm{k}, \mathrm{L}\right)}{\partial \mathrm{Q} \partial \mathrm{k}}=\frac{\partial^{2} \operatorname{EACD}\left(\mathrm{Q}, \pi_{\mathrm{x}}, \mathrm{k}, \mathrm{L}\right)}{\partial \mathrm{k} \partial \mathrm{Q}}$

$=\frac{\mathrm{D}}{\mathrm{Q}^{2}}\left[\frac{\beta_{0}}{\pi_{0}} \pi_{\mathrm{x}}^{2}+\pi_{0}-\beta_{0} \pi_{\mathrm{x}}\right] \sigma \sqrt{\mathrm{L}}\left(1-\frac{\mathrm{k}}{\sqrt{1+\mathrm{k}^{2}}}\right)$

Next, we proceed to evaluate the principal minor of $\mathrm{H}$ at point $\left(\mathrm{Q}^{*}, \pi_{\mathrm{x}}{ }^{*}, \mathrm{k}^{*}\right)$. The first principal minor of $\mathrm{H}$ is:

$\left|\mathrm{H}_{11}\right|$

$=\frac{2 \mathrm{AD}}{\mathrm{Q}^{* 3}}+\frac{\mathrm{D}}{\mathrm{Q}^{* 3}}\left[\frac{\beta_{0}}{\pi_{0}} \pi_{\mathrm{x}}^{* 2}+\pi_{0}-\beta_{0} \pi_{\mathrm{x}}^{*}\right] \sigma \sqrt{\mathrm{L}}\left(\sqrt{1+\mathrm{k}^{* 2}}-\mathrm{k}^{*}\right)$

$+\frac{2 D}{Q^{* 3}}\left[a_{i}\left(L_{i-1}-L\right)+\sum_{j=1}^{i-1} a_{j}\left(T_{j}-t_{j}\right)\right]>0$

The second principal minor of $\mathrm{H}$ is:

$$
\begin{aligned}
& \left|\mathrm{H}_{22}\right|=\frac{2 \beta_{0} \mathrm{D}^{2} \sigma \mathrm{L}^{1 / 2}\left(\sqrt{1+\mathrm{k}^{* 2}}-\mathrm{k}^{*}\right)}{\mathrm{Q}^{* 4} \pi_{0}} \\
& \left\{\mathrm{~A}+\left[\mathrm{a}_{\mathrm{i}}\left(\mathrm{L}_{\mathrm{i}-1}-\mathrm{L}\right)+\sum_{\mathrm{j}=1}^{\mathrm{i}-1} \mathrm{a}_{\mathrm{j}}\left(\mathrm{T}_{\mathrm{j}}-\mathrm{t}_{\mathrm{j}}\right)\right]\right\} \\
& +\frac{\beta_{0} D^{2} \sigma^{2} L\left(\sqrt{1+k^{* 2}}-k^{*}\right)^{2}}{Q^{* 4}}\left(1-\frac{\beta_{0}}{4}\right)>0
\end{aligned}
$$

By substituting $\pi_{\mathrm{x}}{ }^{*}$ from (9) and $\left(\mathrm{k} /\left(1+\mathrm{k}^{* 2}\right)^{1 / 2}\right)$ from (11), the third principal minor of $\mathrm{H}$ is:

$$
\begin{aligned}
& \left|\mathrm{H}_{33}\right|=\left\{\begin{array}{l}
\frac{2 \beta_{0} \mathrm{D}^{2} \sigma \mathrm{L}^{1 / 2}\left(\sqrt{1+\mathrm{k}^{* 2}}-\mathrm{k}^{*}\right)}{\mathrm{Q}^{4} \pi_{0}} \\
\left.\mathrm{~A}+\left[\mathrm{a}_{\mathrm{i}}\left(\mathrm{L}_{\mathrm{i}-1}-\mathrm{L}\right)+\sum_{\mathrm{j}=1}^{\mathrm{i}-1} \mathrm{a}_{\mathrm{j}}\left(\mathrm{T}_{\mathrm{j}}-\mathrm{t}_{\mathrm{j}}\right)\right]\right\} \\
+\frac{\beta_{0} \mathrm{D}^{2} \sigma^{2} \mathrm{~L}\left(\sqrt{1+\mathrm{k}^{* 2}}-\mathrm{k}^{*}\right)^{2}}{\mathrm{Q}^{4}}\left(1-\frac{\beta_{0}}{4}\right)
\end{array}\right\} \\
& \times \frac{1}{2}\left[\begin{array}{l}
\mathrm{h}\left(1-\frac{\beta_{0}}{\pi_{0}} \pi_{\mathrm{x}}^{*}\right) \\
+\frac{\mathrm{D}}{\mathrm{Q}^{*}}\left(\frac{\beta_{0}}{\pi_{0}} \pi_{\mathrm{x}}^{* 2}+\pi_{0}-\beta_{0} \pi_{\mathrm{x}}^{*}\right)
\end{array}\right] \sigma \sqrt{\mathrm{L}\left(1+\mathrm{k}^{* 2}\right)^{-3 / 2}} \\
& -\left[\frac{\mathrm{D}}{\mathrm{Q}^{* 2}}\left[\frac{\beta_{0}}{\pi_{0}} \pi_{\mathrm{x}}^{* 2}+\pi_{0}-\beta_{0} \pi_{\mathrm{x}}^{*}\right] \sigma \sqrt{\mathrm{L}}\left(1-\frac{\mathrm{k}^{*}}{\sqrt{1+\mathrm{k}^{* 2}}}\right)\right]^{2} \\
& \times \frac{2 \mathrm{D} \beta_{0}}{\mathrm{Q}^{*} \pi_{0}} \sigma \sqrt{\mathrm{L}}\left(\sqrt{1+\mathrm{k}^{* 2}}-\mathrm{k}^{*}\right)
\end{aligned}
$$

$$
\begin{aligned}
& \times\left[\begin{array}{l}
\mathrm{h}\left(1-\frac{\beta_{0}}{\pi_{0}} \pi_{\mathrm{x}}^{*}\right) \\
+\frac{\mathrm{D}}{\mathrm{Q}^{*}}\left(\frac{\beta_{0}}{\pi_{0}} \pi_{\mathrm{x}}^{* 2}+\pi_{0}-\beta_{0} \pi_{\mathrm{x}}^{*}\right)
\end{array}\right] \sigma \sqrt{\mathrm{L}}\left(1+\mathrm{k}^{* 2}\right)^{-3 / 2} \\
& {\left[\begin{array}{l}
\mathrm{h}\left(1-\frac{\beta_{0}}{\pi_{0}} \pi_{\mathrm{x}}^{*}\right) \\
+\frac{\mathrm{D}}{\mathrm{Q}^{*}}\left(\frac{\beta_{0}}{\pi_{0}} \pi_{\mathrm{x}}^{* 2}+\pi_{0}-\beta_{0} \pi_{\mathrm{x}}^{*}\right)
\end{array}\right] \sigma \sqrt{\mathrm{L}}\left(1+\mathrm{k}^{* 2}\right)^{-3 / 2}} \\
& +\frac{\beta_{0} \mathrm{D}^{2} \sigma^{2} \mathrm{~L}\left(\sqrt{1+\mathrm{k}^{* 2}}-\mathrm{k}^{*}\right)^{2}}{8 \mathrm{Q}^{* 4}}\left(4-\beta_{0}\right) \\
& \times \mathrm{h}\left(1-\frac{\beta_{0}}{\pi_{0}} \pi_{\mathrm{x}}^{*}\right) \times \sigma \sqrt{\mathrm{L}}\left(1+\mathrm{k}^{* 2}\right)^{-3 / 2} \\
& +\frac{\mathrm{D}^{3} \sigma^{2} \mathrm{~L}}{8 \mathrm{Q}^{* 5}} \sigma \sqrt{\mathrm{L}}\left(\sqrt{1+\mathrm{k}^{* 2}}-\mathrm{k}^{*}\right) \\
& {\left[\frac{\beta_{0} \pi_{0}}{4}\left(\frac{\mathrm{hQ}}{\pi_{0} \mathrm{D}}\right)^{2}+\pi_{0}\left(1-\frac{\beta_{0}}{4}\right)\right] \times \mathrm{G}\left(\mathrm{k}^{*}\right)}
\end{aligned}
$$

where

$$
\begin{aligned}
& \mathrm{G}\left(\mathrm{k}^{*}\right)= \beta_{0}\left(4-\beta_{0}\right) \times\left(1+\mathrm{k}^{* 2}\right)^{-\frac{3}{2}} \times\left(\sqrt{1+\mathrm{k}^{* 2}}-\mathrm{k}^{*}\right) \\
&-2 \times\left(1-\frac{\mathrm{k}^{*}}{\sqrt{1+\mathrm{k}^{* 2}}}\right)^{2} \\
& \times\left\{\frac{1}{4} \times\left[\begin{array}{l}
-2\left(\mathrm{k}^{*}+\sqrt{1+\mathrm{k}^{* 2}}\right)^{2}-\beta_{0} \\
+\sqrt{\left(-2\left(\mathrm{k}^{*}+\sqrt{1+\mathrm{k}^{* 2}}\right)^{2}-\beta_{0}\right)^{2}+\beta_{0}\left(4-\beta_{0}\right)}
\end{array}\right]^{2}\right. \\
&\left.+\beta_{0}\left(1-\frac{\beta_{0}}{4}\right)\right\}
\end{aligned}
$$

For $\mathrm{k}^{*} \in[0, \infty)$ and $0<\beta_{0} \leq 1, \mathrm{G}\left(\mathrm{k}^{*}\right)$ is positive. Hence, we have $\left|\mathrm{H}_{33}\right|>0$.

Therefore, from (13) to (15), it is concluded that the Hessian matrix $\mathrm{H}$ is positive define at point $\left(\mathrm{Q}^{*}\right.$, $\left.\pi_{\mathrm{x}}^{*}, \mathrm{k}^{*}\right)$.

\section{NOTATION AND ASSUMPTIONS}

The following notation is used throughout the study.

$\mathrm{L}=$ Length of lead time (decision variable)

$\mathrm{Q}=$ Order quantity (decision variable)

$\pi_{\mathrm{x}}=$ Backorder price discount offered by the supplier per unit (decision variable)

$\mathrm{k}=$ Safety factor (decision variable)

$\mathrm{r}=$ Reorder point

$\pi_{0}=$ Gross marginal profit per unit

$\mathrm{D}=$ Average demand per year

$\mathrm{A} \quad=$ Fixed ordering cost per order 
Am. J. Applied Sci., 6 (3): 387-392, 2009

$\mathrm{h}=$ Inventory holding cost per unit per year

$\mu \quad=$ Average demand rate in units/day

$\beta=$ Backorder ratio

$\beta_{0}=$ Upper bound of the backorder ratio

$\phi \quad=$ Standard normal distribution

$\Phi=$ Standard normal cumulative distribution function

$\mathrm{SS}=$ Safety stock

$\mathrm{B}(\mathrm{r})=$ Expected shortage of a cycle

$\mathrm{R}(\mathrm{L})=$ Total crashing cost of a cycle

The assumptions made in the study are listed below:

- The reorder point $\mathrm{r}=$ expected demand during lead time + safety stock, that is, $\mathrm{r}=\mathrm{DL}+\mathrm{k} \sigma \sqrt{\mathrm{L}}$, where $\mathrm{k}$ is safety factor

- The lead time $\mathrm{L}$ has $\mathrm{n}$ mutually independent components. The ith component has a normal duration $\mathrm{T}_{\mathrm{i}}$ and a minimum duration $\mathrm{t}_{\mathrm{i}}, \mathrm{i}=1,2, \ldots$, $\mathrm{n}$ and a crashing cost per unit time $\mathrm{a}_{\mathrm{i}}$. The $\mathrm{a}_{\mathrm{i}}{ }^{\prime} \mathrm{s}$ are arranged such that $\mathrm{a}_{1} \leq \mathrm{a}_{2} \leq \ldots \leq \mathrm{a}_{\mathrm{n}}$. The lead times are crashed one component at a time starting with the one of least $c_{i}$ and so on

- Let $\mathrm{L}_{\mathrm{i}}$ denote the length of lead time with component $1,2, \ldots, \mathrm{i}$ crashed to their minimum values, then $L_{i}$ can be expressed as $L_{i}=\sum_{j=1}^{n} T_{j}-$ $\sum_{\mathrm{j}=1}^{\mathrm{i}}\left(\mathrm{T}_{\mathrm{j}}-\mathrm{t}_{\mathrm{j}}\right)$. Thus, the lead time crashing cost $R(L)$ per replenishment cycle is given by $R(L)=a_{i}$ $\left(\mathrm{L}_{\mathrm{i}-1}-\mathrm{L}\right)+\sum_{\mathrm{j}=1}^{\mathrm{i}-1} \mathrm{a}_{\mathrm{j}}\left(\mathrm{T}_{\mathrm{j}}-\mathrm{t}_{\mathrm{j}}\right)$, for $\mathrm{L} \in\left(\mathrm{L}_{\mathrm{i}}, \mathrm{L}_{\mathrm{i}-1}\right]^{[16]}$

- The backorder ratio $\beta$ is variable and is in proportion to the backorder price discount offered by the supplier per unit $\pi_{x}$, thus, $\beta=\beta_{0} \pi_{x} / \pi_{0}$, for $0<\beta_{0} \leq 1,0 \leq \pi_{\mathrm{x}} \leq \pi_{0}^{[16]}$

\section{REFERENCES}

1. Blackburn, J.D., 1991. Time-Based Competition: The Next Battleground in American Manufacturing. Richard Irwin, Inc., Homewood, Illinois.

2. Ben-Daya, M. and A. Raouf, 1994. Inventory models involving lead time as a decision variable. J. Operat. Res. Soc., 45: 579-582.

3. Gallego, G. and I. Moon, 1993. The distribution free newsboy problem, review and extensions. J. Operat. Res. Soc., 44: 825-834.

4. Hariga, M.A. and M. Ben-Daya, 1999. Some stochastic inventory models with deterministic variable lead time. Eur. J. Operat. Res., 113: 42-51.

5. Liao, C.J. and C.H. Shyu, 1991. Analytical determination of lead time with normal demand. Int. J. Operat. Res. Prod. Manage., 11: 72-78.
6. Liao, C.J., 1992. Optimal control of jobs for production systems. Comput. Ind. Engine, 22: 163-169.

7. Moon, I. and S. Choi, 1995. The distribution free newsboy problem with balking. J. Operat. Res. Soc., 46: 537-542.

8. Moon, I. and S. Choi, 1997. Distribution free procedures for Make-To-Order (MTO), Make-InAdvance (MIA) and composite policies. Int. J. Prod. Econ., 48: 21-28.

9. Moon, I. and W. Yun, 1997. The distribution free job control problem. Comput. Ind. Eng., 32: 109-113.

10. Moon, I. and S. Choi, 1998. A note on lead time and distribution assumptions in continuous review inventory models. Comput. Operat. Res., 25: 1007-1012.

11. Moon, I. and E. Silver, 2000. The multi-item newsvendor problem with a budget constraint and fixed ordering costs. J. Operat. Res. Soc., 51: 602-608.

12. Naddor, N., 1966. Inventory System. John Wiley, New York.

13. Lo, M.C., J.C. H. Pan, K.C. Lin and J.W. Hsu, 2008. Impact of lead time and safety factor in mixed inventory models with backorder discounts, J. Applied Sci., 8: 528-533.

14. Ouyang, L.Y., N.C. Yen and K.S. Wu, 1996. Mixture inventory model with backorders and lost sales for variable lead time. J. Operat. Res. Soc., 47: 829-832.

15. Ouyang, L.Y. and K.S. Wu, 1998. A minimax distribution free procedure for mixed inventory model with variable lead time. Int. J. Prod. Econ., 56: 511-516.

16. Pan, J.C. and Y.C. Hsiao, 2001. Inventory models with backorder discounts and variable lead time. Int. J. Syst. Sci., 32: 925-929.

17. Ravindran, A., D.T. Phillips and J.J. Solberg, 1987. Operations Research, Principle and Practices. John Wiley, New York.

18. Silver, E.A. and R. Peterson, 1985, Decision System for Inventory Management and Production Planning. John Wiley, New York.

19. Silver, E., I. Moon and E. Silver, 2001. The multiitem single period problem with an initial stock of convertible units. Eur. J. Operat. Res., 132: 466-477.

20. Tersine R.J., 1994. Principles of inventory and material management. North Holland, New York.

21. Vollmann, T.E., W.L. Berry and D.C. Whybark, 1992. Manufacturing Planning and Control System. 3rd Edn. Irwin, Chicago. 